\title{
Alcohol Sensitivity and Smoking History in Men and Women
}

\author{
P. A. F. Madden, A. C. Heath, G. A. Starmer, J. B. Whitfield, and N. G. Martin
}

\begin{abstract}
Many studies have found genetic effects to contribute to alcoholism risk in both men and women. Based on preliminary evidence for shared genetic risk between smoking and drinking problems, a reanalysis of alcohol challenge data on $\mathbf{4 1 2}$ Australian twins was performed to explore the possibility that smoking may diminish or moderate the intoxicating effects of alcohol. We found history of smoking to be strongly associated with self-reported intoxication after alcohol challenge in women (women: $r=-0.44 \pm 0.08$; men: $r=-0.21 \pm$ 0.08 ), comparable with self-reported average weekly consumption of alcohol, which was more strongly associated in men (women: $r=$ $-0.37 \pm 0.07$; men: $r=-0.54 \pm 0.06$ ). Structural equation modelfitting indicated a strong association between heavy drinking and smoking, but the association between smoking and postalcohol intoxication remained even when the effects of heavy drinking were controlled for. These results prompt the question of whether smoking cigarettes directly influences the transition from moderate to excessive use of alcohol by diminishing feelings of alcohol intoxication.
\end{abstract}

Key Words: Alcohol Intoxication, Alcohol Challenge, Smoking.

$\mathbf{F}$ INDINGS FROM twin and adoption studies are consistent with an important genetic contribution to alcoholism risk in men, and at least some studies suggest a genetic influence on alcoholism in women (reviewed in refs. 1 and 2). A relationship between smoking tobacco and the excessive use of alcohol has been recognized for many years. ${ }^{3}$ Recently, a population-based study found those who have ever smoked to have five times the risk of developing alcoholism as compared with nonsmokers. ${ }^{4}$ A series of twin studies of smoking behavior yielded results in both men and women consistent with an important genetic influence on various aspects of smoking: whether or not an individual becomes a smoker, how much she or he smokes, and whether or not he or she quits successfully or becomes a persistent long-term smoker. ${ }^{5-12}$ It is therefore natural to

From the Department of Psychiatry (P.A.F.M., A.C.H.), Washington University School of Medicine, Medical School, St. Louis, Missouri; Psychopharmacology Research Unit (G.A.S.), Department of Pharmacology, University of Sydney, Sydney, Australia; Department of Biochemistry (J.B.W.), Royal Prince Alfred Hospital, Sydney, Australia; and Queensland Institute of Medical Research (N.G.M.), Brisbane, Queensland, Australia.

Received for publication July 26, 1994; accepted March 28, 1995

This study was supported in part by the National Institutes of Health Grants AA07535 and AA07728, by Postdoctoral Training Grant DA07261, by a grant from the Australian NH\&MRC, and by a grant from the Australian Associated Brewers.

Reprint requests: Pamela A. F. Madden, Ph.D., Department of Psychiatry, Washington University School of Medicine, 4940 Children's Place, St. Louis. MO 63110.

Copyright $(\subseteq) 1995$ by The Research Society on Alcoholism.

Alcohol Clin Exp Res. Vol 19, No 5, 1995: pp 1111-1120 ask whether there is an association between smoking and the genetic contribution to alcoholism risk. Swan et al. ${ }^{9}$ reported a significant genetic association between smoking and level of alcohol consumption in U.S. Veteran twins, but they did not assess problems with alcohol. A preliminary analysis of the relationship between cigarette smoking and questionnaire data on problems related to alcohol has been performed by Madden et al. ${ }^{13}$ in two cohorts of Australian twins. Findings suggest that smoking initiation and the development of problems with alcohol share genetic risk factors in common, and that some of the genetic risk shared may be independent from both of these measures and personality, especially in women. ${ }^{13}$ These latter two studies raise questions about the causal relationship between smoking cigarettes, heavy drinking, and later problems with alcohol.

\section{GENETICS AND ALCOHOL SENSITIVITY}

Behavioral and physiological responses to challenge with an acute dose of alcohol have been systematically examined in offspring (usually sons) of alcoholics and controls in an effort to identify markers of susceptibility to alcoholism. The behavioral responses most often reported to discriminate individuals by family history of alcoholism are body sway ${ }^{14-17}$ and feelings of subjective intoxication. ${ }^{18-24}$ Most high-risk studies of alcohol challenge performance have assumed, at least implicitly, a strong genetic determination of differences in postalcohol performance. Results from an alcohol challenge study conducted on a sample of Australian twins ${ }^{25,26}$ confirm that a substantial portion of the postalcohol variation in body sway ${ }^{25}$ and self-report of intoxication ${ }^{27}$ can be attributed to genetic effects. A metaanalysis of self-rated measures of intoxication ${ }^{28}$ supports the premise that sons of alcoholics experience less intense intoxication in response to an acute dose of alcohol, and this is consistent with data from a 10-year follow-up of challenge study participants ${ }^{29,30}$ that suggest that decreased ratings of self-intoxication measured in young men may predict alcohol dependence in later adulthood.

\section{SMOKING AND ALCOHOL SENSITIVITY}

Nicotine and alcohol have opposing effects on certain functions important to information processing. Findings from experimental studies on the effects of alcohol and nicotine consumed both separately and jointly suggest that 
nicotine counteracts acute effects of alcohol on cognitive skills, such as alertness (arousal) and speed of decisionmaking. ${ }^{31-34}$ Furthermore, preliminary evidence suggests that stimulant effects of nicotine may actually be enhanced when smoking occurs, together with low levels of alcohol consumption. ${ }^{32}$

We know of no detailed investigation of the relationship between smoking and alcohol sensitivity in humans. However, a substantial literature on this relationship in rodents is emerging. A systematic examination of genetic regulation of initial sensitivity to alcohol and nicotine and the development of tolerance has been initiated by Collins et al. who have found that mouse strains selectively bred for alcohol sensitivity also display differences in sensitivity to nicotine. ${ }^{35,36}$ They have observed a strong trend for similar characteristics in rat lines at an earlier stage in selective breeding. ${ }^{37,38}$ Because differences have not been found in nicotine distribution or metabolism between the sensitive and more resistant strains, variation in drug response is thought to be caused by CNS sensitivity. ${ }^{38,39}$ A preliminary study has shown chronic treatment with ethanol in mice to be associated with significant decreases in sensitivity to an acute dose of nicotine, and, interestingly, nicotine desensitization after a pretreatment dose of nicotine has been observed to be greater when given together with a dose of alcohol. Less evidence has been found for ethanol crosstolerance, which has been demonstrated only in mice with initially high levels of drug sensitivity. ${ }^{39,40}$

Findings in Australian twins that suggest shared genetic risk between smoking and drinking problems, together with evidence for cross-tolerance to nicotine and atcohol from rodent strains, prompt the question of whether the additive or interactive effects of nicotine and alcohol play an important role in the development of tolerance to alcohol in humans and, more generally, in the development of alcohol problems. Herein, we address the preliminary question of whether the joint use of nicotine and alcohol moderates alcohol response in humans by performing a reanalysis of alcohol challenge data that includes information on women as well as men, from the Australian alcohol challenge twin study. ${ }^{25,26}$ The present analyses sought to examine the following questions: Is smoking history a predictor of alcohol challenge performance, with history of smoking predicting decreased intoxication after a body weight-adjusted dose of alcohol? If so, are there effects of smoking history that are additional to or that enhance the effects of previous experience with alcohol on alcohol challenge performance?

\section{METHODS}

Sample

The sample was comprised of male and female adult volunteers recruited from the Australian NH \& MRC Twin Registry by one of the authors (N.G.M.) in 1979. Two hundred thirteen female and 199 male twins of European descent, with a mean age of 23 years (range 18-34 years), completed the study. A small number of volunteers were excluded from the study because their cotwin refused to participate or complete the experimental protocol, or when either twin was found to be a lifetime abstainer from alcohol. Furthermore, subjects were dismissed if alcohol was present in their blood at the time of baseline testing. Fewer than $5 \%$ of the twins accepted into the study did not complete the protocol, and this

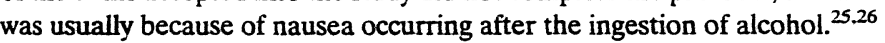
Subjects were not screened for personal or family history of alcoholism. However, the laboratory subjects reported drinking less frequently, but more heavily than did Australian twin volunteers of the same ages from a much larger sample (men: 1961; female: 3121) surveyed in 1981, 2 years after the experimental study (see ref. 41 for a discussion of the representativeness of the 1981 sample with respect to alcohol consumption variables). $13.3 \%$ of the alcohol challenge study women vs. $14.8 \%$ of the women from the questionnaire survey and $18.9 \%$ vs. $31.7 \%$ of the men reported drinking every day or most days, whereas $45.4 \%$ vs. $27.1 \%$ of the women and $64.1 \%$ vs. $56.2 \%$ of the men reported taking six or more drinks on average/occasion (unpublished data). The proportion of smokers among the laboratory subjects was somewhat greater, compared with numbers found among the 1981 survey sample wherein only $44.5 \%$ of the women and $48.5 \%$ of the men reported smoking cigarettes sometime in their life (current smokers-women: $29.5 \%$; men: $30.6 \%$ ). ${ }^{42} 52.1 \%$ of the women among the laboratory sample and $56.1 \%$ of the men reported lifetime smoking; $38.5 \%$ of the women and $36.4 \%$ of the men identified themselves as current smokers. The overrepresentativeness of heavy drinkers is not unexpected, given that subjects were volunteering for an experiment involving the ingestion of alcohol. Well-educated individuals were somewhat overrepresented in the sample; $>60 \%$ of the laboratory subjects had achieved $>12$ years of education, but a broad range of educational and socioeconomic levels were represented. Deletion of cases with missing data reduced the final sample to 191 men and 207 women for the analyses presented herein.

\section{Measures}

Before laboratory procedures were begun, each subject completed a questionnaire that obtained estimates of drinking history, including frequency of consumption (every day or most days; a couple of times a week; once every week or so; or very rarely); a lifetime estimate of the number of occasions alcohol has been consumed (10 times or less; between 11 and 50 times; between 51 and 100 times; between 101 and 500 times; between 501 and 1000 times; or $>1000$ times); the average number of drinks consumed/week [wherein the number of glasses of beer, cider, wine, fortified wine, or spirits reported were summed and this value $(x)$ transformed by taking $\log (x+1)$ ]; and heavy drinking [i.e., number of drinks consumed on average/occasion ( 9 or more, $6-8,3-5$, or 2 or less drinks)], frequency hungover, and frequency drunk (with the last two variables rated on three levels: often, sometimes, or never). Information was also obtained on smoking history. Subjects endorsed 1 of 3 options to the question, "Are you: (1) a current smoker; (2) past smoker; or (3) nonsmoker?" For analyses presented herein, we use two classifications of smoking status: current smoking (current versus nonsmoker or past smoker) and ever smoked (current or past versus nonsmoker).

The experimental protocol included a battery of psychomotor tests and ratings of subjective intoxication. For the purposes of this study, we reexamined data on two measures previously demonstrated to discriminate most clearly between sons of alcoholics and controls after a standard dose of alcohol, body sway, and self-ratings of intoxication. ${ }^{14,19,28}$ Body sway was measured by having subjects, under instructions to remain relaxed and as steady as possible, stand on a platform with a displacement transducer mounted underneath that translated any forward-backward shift in position into an electrical impulse. These impulses were recorded on a Grass Polygraph and integrated to quantify body sway under two conditions: eyes open and eyes closed. Measures were taken more than once and averaged to compute a score that was then adjusted for height (further details in ref. 25). More negative scores indicated increased body sway. Feelings of intoxication were measured by having subjects rate, "How do you feel now?", on a scale from 1 to 10 , wherein $1=$ "completely sober" and $10=$ "the most drunk I have ever been." At baseline, all 


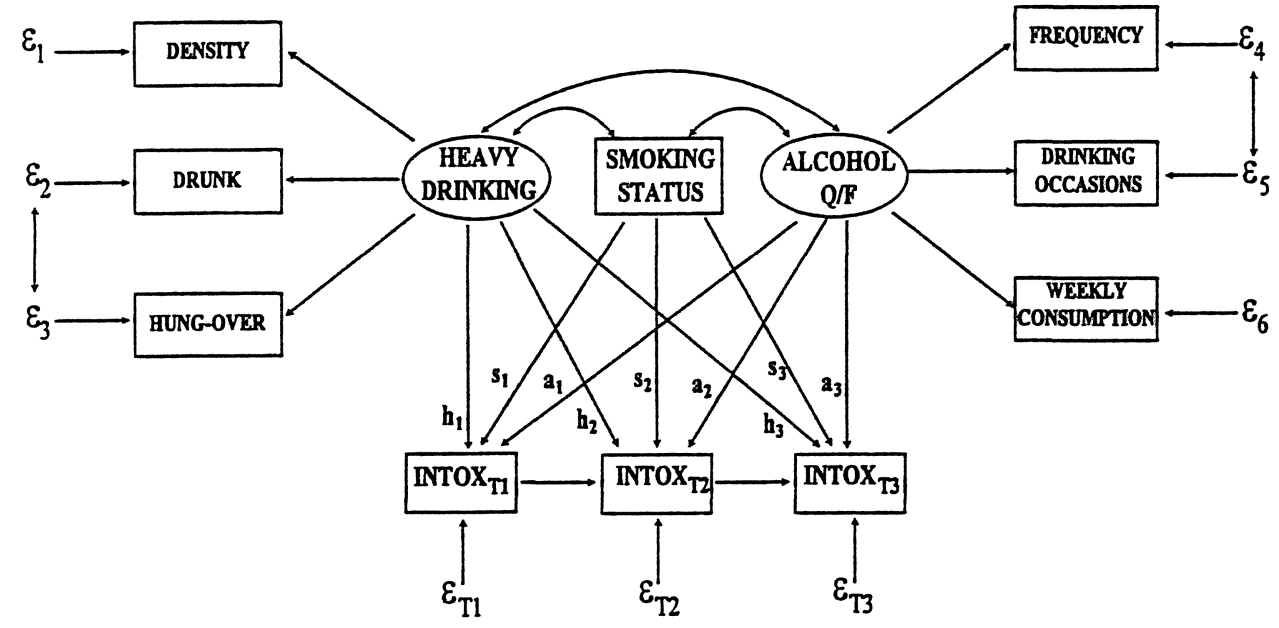

Fig. 1. Depicts the full structural equation model that allows for the effects of smoking history and experience with alcohol on selfratings of intoxication taken during testing cycles begun at 20,80 , and 140 min postalcohol. Rectangles are used to denote observed variables, and ellipses to denote latent variables. $\xi_{1} \ldots \xi_{6}$ and $\xi_{T 1} \ldots \xi_{T 3}$ denote residuals for the measures of drinking history and postalcohol intoxication rating.

subjects rated themselves as completely sober. Subjects were instructed to eat a nonfatty meal $\sim 8: 00 \mathrm{AM}$, and procedures began at 9:00 in the morning. No restrictions were placed on smoking, and subjects were permitted to smoke as they wished throughout the experimental protocol. After completing the baseline questionnaire and baseline psychomotor tasks, subjects were asked to drink an alcohol dose of $0.75 \mathrm{~g}$ ethanol $/ \mathrm{kg}$ body weight diluted to $10 \%(\mathrm{v} / \mathrm{v})$ in a sugarless "lemon squash" (a mixture of lemon and soda water) within $20 \mathrm{~min}$ at a steady rate. No placebo control group was tested, nor were responses taken at other doses of alcohol. Six blood alcohol levels were taken during and between testing sessions; the first was drawn after a battery of tests given at $20 \mathrm{~min}$ after the ingestion of alcohol and the others at regular intervals over $3.5 \mathrm{hr}$ (mean sampling times: $57,70,85,125,145$, and $185 \mathrm{~min}$ postalcohol). The battery of psychomotor tests was administered at 20,80, and $140 \mathrm{~min}\left(t_{1}\right.$, $t_{2}$, and $t_{3}$ ) after alcohol intake, over a 25-min testing cycle. Blood alcohol levels and intoxication ratings were obtained at the end of each testing cycle, with additional blood alcohol level assessments made between cycles. Testing procedures are described more fully in Martin et al. ${ }^{25,26}$ No restrictions were placed on smoking. However, they would have had somewhat limited opportunities to smoke during each 25-min testing cycle. Unfortunately, smoking by subjects during the protocol was undocumented.

\section{DATA ANALYSIS}

\section{Preliminary Analyses}

Blood alcohol levels, subjective intoxication ratings, and body sway (both eyes open and eyes closed conditions) were plotted as a function of smoking status, separately for men and women. Polychoric and polyserial correlations between first postalcohol assessments of these variables (20 min after alcohol ingestion) and measures of smoking status and drinking history were estimated using PRELIS. ${ }^{43}$ Only subjective intoxication showed a strong association with smoking status in both sexes; therefore, after the preliminary analyses of these data, all additional analyses reported were performed using this variable.

\section{Structural Equation Modeling}

To evaluate further the relationship between postalcohol intoxication ratings, smoking, and drinking history measures, we fitted the linear structural model illustrated in
Fig. 1, using LISREL VII. ${ }^{44}$ Fitting LISREL models may be viewed as a generalization of conventional regression analysis, which allows us to handle multiple measures of our independent (predictor) variables, and multiple dependent variables that have a time-series structure (see ref. 45 for a detailed discussion of structural equation modeling). The dependent variables were self-ratings of intoxication taken after a battery of psychomotor tests administered at three points of time $(20,80$, and $140 \mathrm{~min})$ after ingestion of alcohol. Because the correlations between these sequential measures demonstrated a pattern of decreasing association for measures taken at more distant points in time, the relationships among these variables were defined in the model as first-order autoregressive. Models with one versus two alcohol consumption latent variables were compared by likelihood-ratio $\chi^{2}$. The model with two latent alcohol consumption variables gave a better fit to these data (women: $p<0.01$; men: $p<0.001$ ). As shown in Fig. 1 , the model used to evaluate the relationship between postalcohol intoxication ratings, smoking, and drinking history measures included three predictor variables (where "predictor" herein is used in a statistical rather than causal sense): a measure of smoking status (in separate analyses, either current smoking or ever smoked) and two alcohol consumption latent variables, each assessed by three indicator variables-(1) ALCOHOL $\mathrm{Q} / \mathrm{F}$ (quantity/frequency) indexed by average weekly consumption (WEEKLY CONSUMPTION), frequency of consumption (FREQUEN$\mathrm{CY}$ ), and lifetime estimate of the number of occasions alcohol has been consumed (DRINKING OCCASIONS); and (2) HEAVY DRINKING indexed by the number of drinks typically consumed/occasion (DENSITY), frequency drunk (DRUNK), and frequency hungover (HUNGOVER). We allowed for correlated measurement errors for the pairs of variables DRUNK and HUNGOVER, and FREQUENCY and DRINKING OCCASIONS, because models that assumed independent errors for all predictor variables generated negative error variance estimates for some variables in some analyses. 
Under the full model, predictor variables had direct paths to subjective intoxication at each of the three time points (paths $s_{1}-s_{3}, h_{1}-h_{3}$, and $a_{1}-a_{3}$ ). We examined whether the effects of drinking were entirely mediated through subjective intoxication at time 1 by testing the fit of models with paths dropped from the two alcohol consumption variables (paths $h_{2}, h_{3}, a_{2}$, and $a_{3}$ ) to subjective intoxication measured during testing cycles begun at 80 and 140 min postalcohol. The effects of smoking were evaluated by testing the fit of models with paths dropped from smoking status to these same two variables (paths $s_{2}$ and $s_{3}$, with paths $h_{2}, h_{3}$ and $a_{2}, a_{3}$ retained in the model). Because the influence of smoking on intoxication was under examination, the effects of smoking behavior were assessed separately from the effects of alcohol consumption. The test for direct effects of smoking on perceived intoxication (independent from level of exposure to alcohol) was completed by dropping the path(s) from smoking status to intoxication while retaining the corresponding paths from the alcohol consumption variables. Last, the presence of collinearity among the three predictor variables was assessed in the reduced model (with deletion of paths $s_{2}, s_{3}, h_{2}, h_{3}$ and $a_{2}$, $a_{3}$ if these were nonsignificant) by alternately eliminating the path from each drinking experience variable to subjective intoxication (paths $h_{1}$ and $a_{1}$ ). Because most of our observed variables were discontinuous, a matrix of polychoric, polyserial, and product-moment correlations was computed using PRELIS, and models were fitted by asymptotic weighted least squares. ${ }^{43,44}$

The usual assumption of independence of observations is violated in twin pair data. The observations on one twin are correlated with the observations on the cotwin. Because sample sizes in this study are small ( $\sim 40$ twin pairs/zygosity group), the number of variables large $(n=10 /$ twin), and some of the variables categorical, it would not be feasible to analyze the data by twin pair to correct for this nonindependence. Nonindependence of the observations will not bias parameter estimates, but does complicate the interpretation of statistical tests of significance. ${ }^{46}$ There is no completely satisfactory solution to this problem available. Therefore, statistical tests were performed using first and second twins as quasireplicate samples, giving us two sets of data on both men and women to evaluate the robustness of our results. Models were compared with the full model of Fig. 1, and reduced models, by likelihood-ratio $\chi^{2}$ to identify the most parsimonious models consistent with the observed data. ${ }^{47}$ Separate parameter values were estimated for each subsample, and when discrepancies occurred over the fit of a particular model between first and second twins, the sum of the $\chi^{2}$ s derived from these two subsamples was used in supplement to guide the model-fitting process. This procedure is somewhat conservative and may cause us to retain in our model parameters that might in fact be nonsignificant. Finally, once the most parsimonious model had been identified, parameter estimates under that model were obtained using all available information (i.e., data from the subsamples were analyzed jointly in a two-group LISREL analysis that constrained parameter values to be the same in the two groups). Separate analyses were conducted by gender.

\section{Testing for Interaction Effects}

We anticipated that there might be an interaction between current smoking and alcohol consumption history, similar to that observed in rodents, such that the effects of drinking history on sensitivity to alcohol was increased in smokers compared with nonsmokers. It is possible in principle to test for such an interaction effect using LISREL, in a multigroup analysis, where current smokers, past smokers, and nonsmokers are included as separate groups, and the homogeneity across groups of the paths from alcohol consumption to intoxication variables is tested by likelihood-ratio $\chi^{2}$ test. In practice, however, the correlations between smoking and alcohol consumption variables were too strong to permit such an approach, and lead to substantial within-group restriction of variability. Therefore, we performed conventional regression analyses to test for a significant interaction effect of alcohol consumption (two measures: average weekly consumption or heavy drinking) and smoking on self-ratings of intoxication taken during the testing cycle begun at $20 \mathrm{~min}$ postalcohol. To avoid multiple significance tests, we limited our analyses to just two measures of alcohol consumption, wherein we had the strongest a priori expectation of finding an interaction with smoking. Because of the possibility of a curvilinear relationship between experience with alcohol and feelings of intoxication, we included quadratic and cubic terms for alcohol consumption in our models.

\section{RESULTS}

\section{Mean Effects as a Function of Smoking Status and Sex}

Figure 2 ( $a$ and $b$ ) shows mean values of the six blood alcohol levels drawn postalcohol as a function of current smoking status and sex. No significant differences were found in blood alcohol levels between current versus nonsmoking (never and ex-smokers) women; however, disparities were found in the blood alcohol levels of men at time points $t_{3}-t_{6}$. Smoking men had significantly lower blood alcohol levels at later time points as compared with the non- or ex-smokers, which suggests that ethanol might have been more efficiently eliminated in our sample of male smokers. Figure 2 (c and d) also displays self-ratings of intoxication obtained during testing cycles begun at 20, 80 , and $140 \mathrm{~min}$ postalcohol. Smokers experienced a significantly lower level of intoxication compared with nonsmokers after a standard dose of alcohol, and this was even more pronounced in females than males. Differences observed 


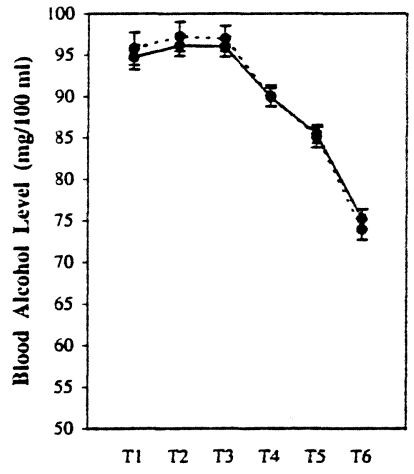

a) WOMEN

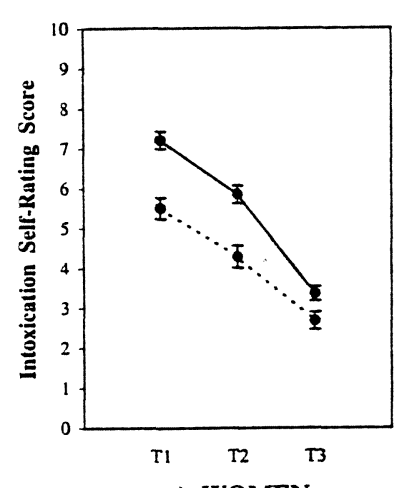

c) WOMEN

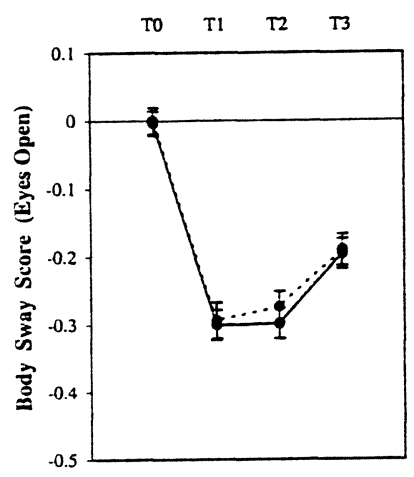

e) WOMEN

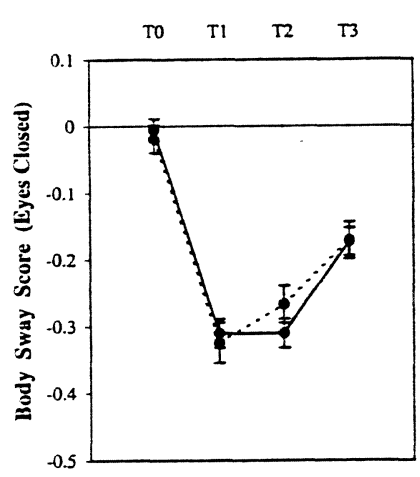

g) WOMEN

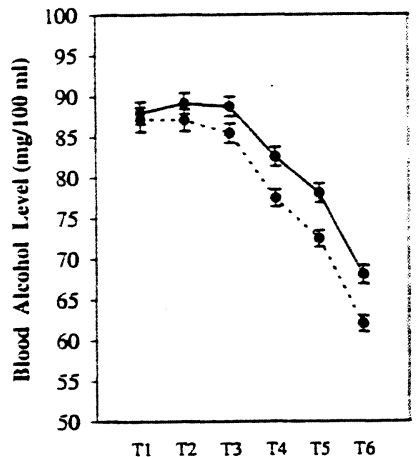

b) MEN

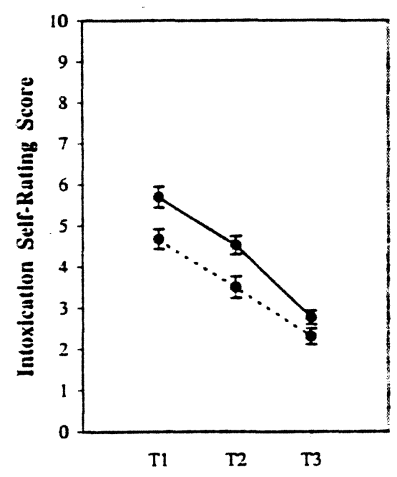

d) MEN
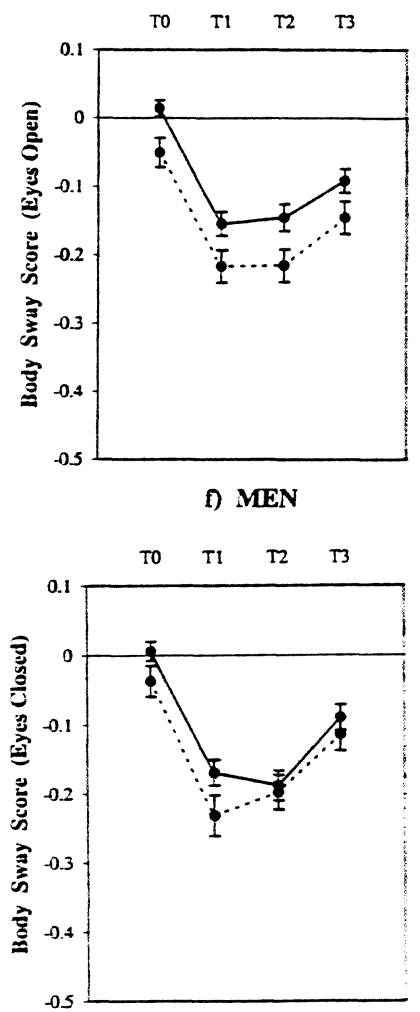

h) MEN
Fig. 2. Mean values and confidence intervals with upper and lower limits = SEM of behavioral and physiological responses to a challenge dose of alcohol in female and male current (- - ) versus nonsmokers [never or ex-smokers (-)]: \{a. b) blood alcohol level $(\mathrm{mg} / 100 \mathrm{ml})$ at 6 points in time drawn at regular intervals over $3.5 \mathrm{hr}$ postalcohol in women (a) and men (b); (c, d) self-ratings of intoxication among ratings of intoxication in our smoking versus non- or ex-smoking subjects were most pronounced at $t_{1}$ and declined thereafter. No significant association between blood alcohol level and self-report intoxication was found in men, and only one significant association, at $t_{3}$, in women $(p=$ $0.02)$. Thus, it does not seem that these differences in perceived intoxication by smoking status can be explained by differences in blood alcohol levels. Figure $2(\mathrm{e}-\mathrm{h})$ shows mean values of body sway with eyes open and eyes closed, measured at baseline as well as $t_{1}-t_{3}$ postalcohol. No significant differences in body sway under either condition was observed in smoking versus nonsmoking women; however, small (but significant) differences were observed under both conditions in men. For body sway measured under both conditions, male smokers seemed to be less steady at baseline and at $t_{1}$ as compared with the non- or ex-smokers; and in the eyes open condition, the smoking males consistently displayed more body sway from baseline through recovery $\left(t_{3}\right)$. Similar patterns were observed for each of these variables in the comparison of the lifetime versus nonsmokers by gender.

\section{Correlates of Smoking Behavior and Self-Ratings of Intoxication}

Smoking status demonstrated a strong correlation with self-ratings of intoxication taken $20 \mathrm{~min}$ postalcohol (current smokers-female: $-0.44 \pm 0.08$; male: $-0.21 \pm 0.08$; ever-smokers-female: $-0.38 \pm 0.08$; male: $-0.29 \pm 0.09$ ), but less association, especially in females, with either measure of body sway taken at the same point in time (current or ever-smokers-females: $-0.01 \pm 0.10$ to $-0.05 \pm 0.11$; males: $-0.05 \pm 0.09$ to $-0.22 \pm 0.09$ ). Average weekly alcohol consumption correlated more highly with ratings of intoxication in men than in women $(-0.54 \pm 0.06$ vs. -0.36 \pm 0.07 ). Smoking status was also highly correlated with history of heavy drinking, especially in females. DENSITY (drinks consumed on a typical occasion) correlated $0.57 \pm$ 0.07 with ever having smoked in women and $0.34 \pm 0.10$ in men (for current smokers-females: $0.59 \pm 0.07$; men: 0.43 \pm 0.08 ). Twenty-five percent (27 of 109) of women who have ever smoked versus $2 \%$ ( 2 of 101) of nonsmokers reported typically taking six or more drinks of alcohol/ occasion, with the corresponding figures in men $26 \%$ (28 of 109 ) and $21 \%$ (18 of 84$)$. DENSITY correlated $-0.39 \pm$ 0.07 with ratings of intoxication in the females and $-0.53 \pm$ 0.06 in the men, showing that even using measures of heavy drinking, current smoking showed no less strong an association with postalcohol intoxication than did experience with drinking in these women.

taken during testing cycles begun at 20,80 . and $140 \mathrm{~min}$ postalcohol in women (c) and men (d); (e, f) measures of body sway taken with eyes open taken at baseline and at $\sim 20,80$, and $140 \mathrm{~min}$ postascohol in women (e) and men (f); and $(\mathrm{g}, \mathrm{h})$ measures of body sway taken with eyes closed taken at baseline and at $-20,80$, and 140 min postajcohol in women (g) and men (h). 


\section{Structural Equation Modeling}

For women, history of smoking and experiences with alcohol seemed to affect level of intoxication measured during the testing cycle begun at 20 min postalcohol, but had no additional direct effect on rate of recovery from intoxication. This was true regardless of whether we examined current smoking (twin 1: $\chi^{2}=4.41, d f=6, p=0.62$; twin 2: $\chi^{2}=5.77, d f=6, p=0.45$ ) or lifetime smoking using data from second twins $\left(\chi^{2}=5.52, d f=6, p=0.45\right)$. Dropping paths from the three predictor variables to intoxication at times two and three (measured during testing cycles begun at 80 and $140 \mathrm{~min}$ postalcohol, respectively) under lifetime smoking for first twins gave a worse fit to the data $\left(\chi^{2}=13.03, d f=6, p=0.04\right)$. However, because the sum of $\chi^{2}$ s provided an insignificant change in $\chi^{2}\left(\chi^{2}=\right.$ $18.55, d f=12, p=0.10$ ), the hypothesis that the effects of smoking and drinking history are entirely mediated through time 1 intoxication $\left(h_{2}=h_{3}=a_{2}=a_{3}=s_{2}=s_{3}=0\right)$ could not be rejected.

For lifetime smoking in women, there was a multicollinearity problem that made it impossible to determine whether there was a direct effect of smoking status on $\mathrm{INTOX}_{11}$ (i.e., dropping any one of the three regression paths from HEAVY, ALCOHOL Q/F, or SMOKING to INTOX $_{11}$ did not lead to a significant worsening of fit to these data). For lifetime smoking, we therefore report parameter estimates under a model, including all three paths to INTOX $_{t 1}$. In contrast, for CURRENT SMOKING, the path from ALCOHOL Q/F to INTOX $_{t 1}$ was clearly nonsignificant (twin 1: $\chi^{2}=0.49, d f=1, p=0.48$; twin 2: $\chi^{2}$ $=0.11, d f=1, p=0.74)$. But dropping out the path from CURRENT SMOKING in addition to the path from ALCOHOL Q/F led to a significant deterioration in fit (twin 1: $\chi^{2}=6.68, d f=1, p<0.01$; twin $2: \chi^{2}=12.16, d f=1, p<$ 0.001); and dropping the path from HEAVY DRINKING in addition to the path from ALCOHOL Q/F led to a significantly worse fit in first twins $\left(\chi^{2}=18.04, d f=1, p<\right.$ $0.001)$, but not in second twins $\left(\chi^{2}=2.77, d f=1, p=0.10\right)$. Again, because the sum of $\chi^{2}$ 's provided a highly significant difference in fit $\left(\chi^{2}=20.81, d f=2, p<0.001\right)$, we retained the paths from CURRENT SMOKING and HEAVY DRINKING to INTOX ${ }_{t 1}$, and dropped only the path from ALCOHOL Q/F. Parameter estimates for women under models determined to best describe the effects of ever becoming a smoker and of current smoking are summarized in Fig. 3 ( $a$ and b). HEAVY DRINKING was very highly correlated with smoking status and somewhat more highly correlated with lifetime smoking (0.92) than with current smoking status (0.79). The estimated correlations between smoking status and ALCOHOL Q/F were somewhat more modest $(0.47,0.67)$. Loadings of DENSITY, DRUNK, and HUNGOVER on the HEAVY DRINKING latent factor (0.54-0.81) were substantial, as were loadings of FREQUENCY, DRINKING OCCASIONS, and WEEKLY CONSUMPTION on the ALCOHOL Q/F fac- tor (0.45-0.77), confirming that these measures were good indices of the underlying constructs. The direct effect of smoking status on postalcohol intoxication was negative for current smokers $(-0.33)$ and for all lifetime (ever) smokers $(-0.51)$.

For EVER SMOKED in men, as for women, we found a direct effect of smoking or drinking history on initial intoxication (INTOX $\mathrm{X}_{11}$ ) taken during the testing cycle begun at $20 \mathrm{~min}$ postalcohol, but no additional direct effect on INTOX $_{t 2}$ or INTOX IN $_{t 3}$ measured during the cycles begun at 80 and $140 \mathrm{~min}$ postalcohol respectively [i.e., no additional effect on rate of recovery from intoxication $\left(a_{2}=a_{3}=h_{2}\right.$ $=h_{3}=s_{2}=s_{3} ;$ twin $1: \chi^{2}=9.65, d f=6, p=0.14$; twin 2 : $\left.\left.\chi^{2}=5.85, d f=6, p=0.44\right)\right]$. In contrast to findings for women, however, the path from EVER SMOKED to INTOX $_{t 1}$ was clearly nonsignificant (twin $1: \chi^{2}=0.39, d f=1$, $p=0.53$; twin $2: \chi^{2}=0.02, d f=1, p=0.89$ ). Dropping out either the path from HEAVY DRINKING or the path from ALCOHOL Q/F in addition to the path from EVER SMOKED led to a significant deterioration in fit in first twins $\left(\chi^{2}=6.33, d f=1, p=0.01 ; \chi^{2}=8.56, d f=1, p<\right.$ $0.01)$, but not in second twins $\left(\chi^{2}=2.21, d f=1, p=0.14\right.$; $\left.\chi^{2}=0.22, d f=1, p=0.64\right)$. However, because the sum of $\chi^{2}$ 's provided significant differences in fit $\left(\chi^{2}=8.54, d f=\right.$ $2, p=0.01 ; \chi^{2}=8.98, d f=2, p=0.01$ ), we retained the paths from HEAVY DRINKING and ALCOHOL Q/F to INTOX $_{t 1}$, and dropped only the path from EVER SMOKED. Parameter estimates for men under the most parsimonious model are summarized in Fig. 3c. The two alcohol consumption variables were strongly correlated (0.83), but each correlated more modestly with lifetime smoking (HEAVY DRINKING: 0.59; ALCOHOL Q/F: 0.50 ). Each alcohol variable had a strong direct effect on initial intoxication (path coefficients -0.30 for HEAVY DRINKING, -0.44 for ALCOHOL Q/F), but there was no significant effect of EVER SMOKED. The indicator variables for HEAVY DRINKING and ALCOHOL Q/F performed even better than for the women, with factor loadings $0.79-0.93$ and $0.85-0.88$, respectively.

For CURRENT SMOKING in men, findings were more complex. The hypothesis of no direct effect of smoking or drinking history on recovery from intoxication (measured during testing cycles begun at 80 and $140 \mathrm{~min}$ postalcohol) was rejected in male first twins $\left(\chi^{2}=15.89, d f=6, p=\right.$ $0.01)$, but was not significant in second twins $\left(\chi^{2}=12.16, d f\right.$ $=6, p=0.06)$. The sum of the $\chi^{2 \text { s, }}$, however, was highly significant $\left(\chi^{2}=28.05, d f=12, p=0.005\right)$. When tested against the full model, dropping the path from CURRENT SMOKING to INTOX ${ }_{12}$ led to a significant worsening of fit in both first and second twins $\left(\chi^{2}=5.91, d f=1, p<0.05\right.$; $\left.\chi^{2}=4.07, d f=1, p=0.04\right)$. This was also true for the path from CURRENT SMOKING to INTOX $_{t 1}$, in first twins $\left(\chi^{2}=8.01, d f=1, p<0.01\right)$, but not second twins $\left(\chi^{2}=\right.$ $0.04, d f=1, p=0.84$ ); however, because the sum of the $\chi^{2}$ s indicated a significant deterioration in fit $\left(\chi^{2}=8.05, d f\right.$ $=2, p=0.02$ ), we retained the path from CURRENT 


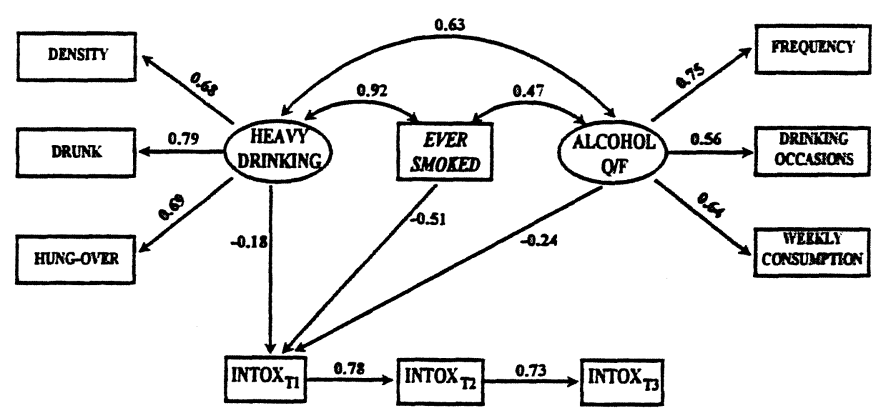

(a) WOMEN

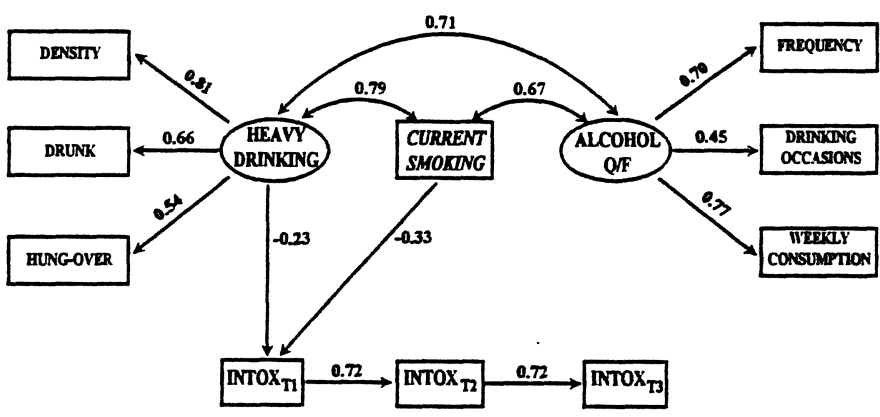

(b) WOMEN

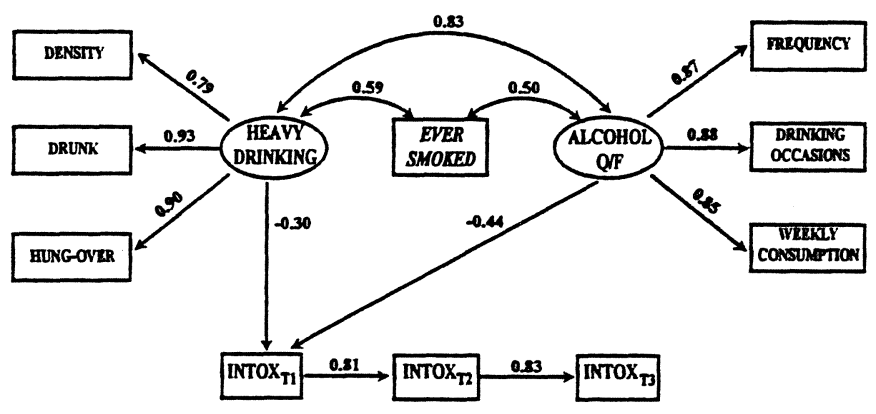

(c) $M E N$

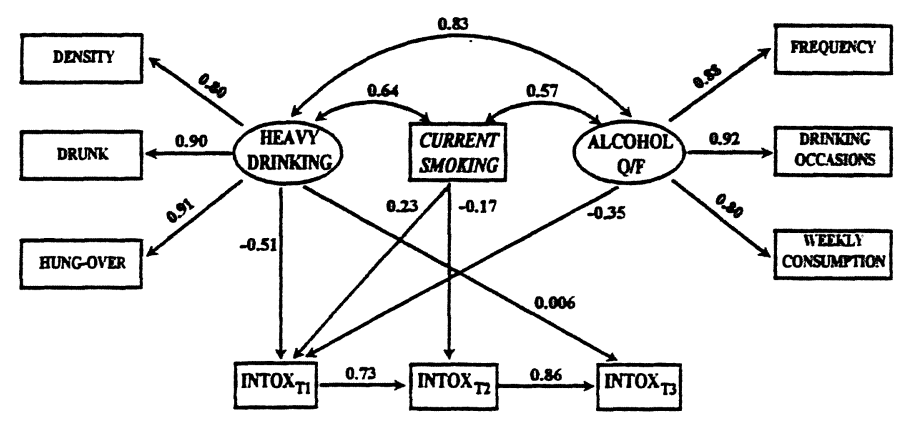

(d) MEN

Fig. 3. Parameter estimates under the best-fitting models in women and men. Error terms have been omitted: (a) female ever versus never smokers; (b) female current versus nonsmokers (never or ex-smokers); (c) male ever versus never smokers; and (d) male current versus nonsmokers (never or ex-smokers).
SMOKING to INTOX $_{t 1}$ as well. The path from CURRENT SMOKING to INTOX ${ }_{t 3}$, however, was nonsignificant (twin 1: $\chi^{2}=0.73, d f=1, p=0.39$; twin $2: \chi^{2}=2.90$, $d f=1, p=0.10$ ).

Deleting paths to INTOX ${ }_{12}$ from HEAVY DRINKING and ALCOHOL Q/F, and to INTOX ${ }_{t 3}$ from ALCOHOL $\mathrm{Q} / \mathrm{F}$, did not significantly worsen the fit of the model (twin 1: $\chi^{2}=4.10, d f=3, p=0.25 ;$ twin $2: \chi^{2}=3.44, d f=3, p$ $=0.33$ ). The path from HEAVY DRINKING to INTOX $_{t 3}$ was nonsignificant in male second twins $\left(\chi^{2}=1.26, d f=1\right.$, $p=0.26)$, but significant in male first twins $\left(\chi^{2}=6.33, d f\right.$ $=1, p=0.01)$; with the sum of the $\chi^{2}$ s significant $\left(\chi^{2}=\right.$ $7.59, d f=2, p<0.05)$, therefore the path from HEAVY DRINKING to INTOX ${ }_{t 3}$ was left in the model. The remaining paths $\left(h_{1}\right.$ and $\left.a_{1}\right)$ could be deleted from the model in male second twins $(p>0.05)$, but not in first twins, nor with the sums of the $\chi^{2}$ s by likelihood-ratio $\chi^{2}$ test $(p<$ 0.05 in all cases). Therefore, all remaining paths were retained.

Figure 3d summarizes parameter estimates for the bestfitting CURRENT SMOKING model in men. Both ALCOHOL $\mathrm{Q} / \mathrm{F}$ and HEAVY DRINKING have strong negative paths to $\operatorname{INTOX}_{t 1}(-0.35,-0.51)$, and the path coefficient from HEAVY DRINKING to INTOX $_{t 3}$ is quite small (0.006). The path to INTOX $_{t 1}$ from CURRENT SMOKING is positive $(0.23)$, indicating that smoking men perceived themselves as more intoxicated during the testing cycle begun at $20 \mathrm{~min}$ postalcohol; but interestingly, there is a negative path from CURRENT SMOKING to INTOX 12 $(-0.17)$ measured during the cycle begun at $80 \mathrm{~min}$ postalcohol. Other parameter estimates are essentially unchanged from the EVER SMOKED analyses.

\section{Testing for Interactive Effects}

In men, main effects on subjective intoxication of heavy drinking, average weekly alcohol consumption, and of ever having smoked were found $(p<0.001, p<0.001$, and $p=$ 0.002 , respectively), as well as a significant interaction between heavy drinking and smoking $(p<0.001)$ in men $\left(R^{2}\right.$ $=0.35)$. The results were similar for current smoking $\left(R^{2}=\right.$ $0.35)$. The effects of ever having smoked and the interaction of smoking with heavy drinking on subjective intoxication remained significant in men under models, including curvilinear terms on both measures of alcohol consumption ( $p=0.01$ and $p=0.01$, respectively; with $R^{2}=0.42$ ), whereas for current smokers only the interaction term remained significant (main effects: $p=0.06$; interaction term: $p=0.04$, with $R^{2}=0.42$ ). To clarify the nature of the significant interaction that we found, the entire sample of men was split into two subgroups by number of drinks consumed on average/occasion (light drinkers: 0-5 drinks; heavy drinkers: 6 or more drinks) to examine the relationship of smoking status to perceived intoxication by level of alcohol consumption. The form of the interaction was not what we had predicted. Among light drinkers, male smok- 
ers had lower mean scores on self-ratings of intoxication as compared with males who had never smoked (mean $\pm \mathrm{SE}$; lifetime smokers vs. never smoked: $4.74 \pm 0.26$ vs. $6.59 \pm$ 0.35 ; current smokers vs. nonsmokers: $4.62 \pm 0.31$ vs. 6.02 \pm 0.29 ). In contrast, among heavy drinkers, it was the smokers who typically perceived themselves as more intoxicated (mean $\pm \mathrm{SE}$; lifetime smokers vs. never smoked: $4.64 \pm 0.34,3.67 \pm 0.42$; current smokers vs. nonsmokers: $4.78 \pm 0.38,3.74 \pm 0.36)$.

In women, the only significant effects were those of drinking experience on perceived intoxication. Apparently, the high correlation of smoking with the measure of heavy drinking was responsible for the nonsignificance of smoking effects in women. When we included only smoking and average weekly consumption as predictors, main effects on subjective intoxication of average weekly alcohol consumption and current smoking was found $(p<0.001$ and $p=$ 0.02 , respectively, with $R^{2}=0.16$ ).

\section{DISCUSSION}

Motivated by preliminary evidence for a significant genetic correlation between smoking and problems with alcohol that was especially strong in women, ${ }^{13}$ we examined the relationship between smoking history and alcohol challenge performance using data from the Australian alcohol challenge twin study. ${ }^{25,26}$ After the ingestion of a standard dose of alcohol, both male and female smokers reported feeling less intoxicated on average than nonsmokers. For women in our sample, smoking status was as associated with perceived intoxication, as was their average weekly alcohol consumption. Male smokers (but not female) also showed increased baseline and postalcohol body sway, and faster recovery of blood alcohol levels to normal. For current smokers, the association between intoxication rating and smoking remained significant when we fitted a structural equation model that also allowed for direct effects of alcohol consumption history on self-report intoxication after alcohol challenge. This result is consistent with our preliminary results on shared genetic risk between smoking and problems with drinking; both raise the possibility that the relationship between smoking and problems with alcohol may not be entirely explained by shared risk-factors such as predisposing personality variables. It is difficult to explain, under such a "shared risk factor" hypothesis, why the association between current smoking and postalcohol intoxication persisted once experiences with alcohol were controlled for (unless, possibly, smoking status is a proxy for personality traits that influence perceptions of intoxication).

Not all studies on sensitivity to alcohol have concluded that men predisposed to alcoholism experience less intoxication $^{48-50}$ or less body sway ${ }^{15,17,20,23,50}$ than men at low risk for drinking problems, and a small number of studies have reported elevated levels of intoxication ${ }^{17,51.52}$ or greater body sway. ${ }^{15,17}$ In addition to differences in exper- imental design and methodology (reviewed in ref. 53), our results suggest that differences in smoking history may be one confound contributing to discrepancies in the alcohol challenge literature. Clearly, it is important that baseline smoking history be taken into account in alcohol challenge research, especially alcohol challenge research with women.

After review of our preliminary analyses, we anticipated finding a significant interaction between smoking history and history of alcohol consumption, such that the decrease in postalcohol intoxication associated with being a current smoker would be substantially greater in heavy drinkers than in light drinkers. In other words, we anticipated that heavy drinking smokers would have acquired much greater chronic tolerance to alcohol. In women, we found that smoking and heavy drinking were very highly confounded (with 27 of 29 of the heaviest drinking women being current or former smokers), so that it was not possible to demonstrate a significant interaction of history of smoking and alcohol consumption. In men, an interaction between having ever smoked and alcohol consumption was found. Among light drinkers, we found that smokers felt less intoxicated than nonsmokers. However, among the heavier drinkers, it was the smokers who reported themselves to be more intoxicated. A similarly unexpected finding emerged from the model-fitting analyses. In women, a negative direct effect was found of current smoking on intoxication self-rating reported during the testing cycle begun at $20 \mathrm{~min}$ postalcohol, as we had anticipated. However, among men, we found that, once we allowed for the effects of heavy drinking in our model, the direct effect of current smoking on intoxication perceived during the first testing cycle after the ingestion of alcohol was positive, indicating that male current smokers were feeling more intoxicated. A negative direct effect of current smoking during the testing cycle begun at 80 min postalcohol was found, suggesting that currently smoking males were showing faster recovery from the effects of alcohol as assessed by subjective intoxication, similar to the finding for blood alcohol levels.

How are these findings to be interpreted? We may speculate either that heavy drinking male smokers habitually smoked, while drinking heavily to regulate their levels of intoxication, and that the initial elevation in intoxication during the first testing cycle (begun $20 \mathrm{~min}$ postalcohol) reflects the fact that these currently smoking males did not use cigarettes as they normally would during this short interval. Thus, they felt more intoxicated than they would normally. Or, alternatively, as previously suggested by Newlin and Thomson, ${ }^{53}$ that individuals with a greater susceptibility to alcohol use experience higher levels of alcohol sensitivity on the ascending part of the blood alcohol curve. ${ }^{54}$ In this case, the heavy drinking male smokers tended to react more strongly after the acute dose of alcohol. Because cigarette use occurring recently before and observed during the experimental protocol was not documented, we were unable to determine the extent to which the effect of smoking on alcohol challenge performance might have been diminished by the differential use 
of tobacco by the current smokers in our sample (i.e., whether smoking has acute effects on alcohol response or whether we are observing chronic effects). We cannot be certain of whether the apparent gender difference is merely a consequence of the very strong association between smoking and heavy drinking in the women from this sample (i.e., a collinearity problem) or a genuine difference.

We had no a priori expectations about whether we would observe the same findings for lifetime smoking as for current smoking. It might be the case that smoking affected the acquisition of chronic tolerance to alcohol, with the effects of the acquired tolerance persisting even after the individual quit smoking. In contrast, if the acute effects of nicotine on postalcohol intoxication were most important, we might expect that current smoking would be the critical variable. For men, we indeed found a significant direct effect only of current smoking; in women, we could not exclude the possibility of a direct effect of lifetime smoking because of the multicollinearity problem.

Further evaluation of the role of smoking in determining alcohol effects is likely to require an experimental approach (e.g., observing the effects of nicotine challenge ${ }^{54}$ and alcohol challenge separately and jointly). The close relationship found between smoking tobacco and excessive alcohol use $\mathrm{e}^{3}$ or risk of alcohol dependence ${ }^{4}$ suggests that this be an important priority for future research.

\section{ACKNOWLEDGMENTS}

We thank Ovide Pomerleau, Ph.D., Allan Collins, Ph.D., and John Hughes, M.D., for helpful comments.

\section{REFERENCES}

1. McGue M: Genes, environment and the etiology of alcoholism, in Development of Alcohol-Related Problems in High-Risk Youth: Establishing Linkages Across Biogenetic and Psychosocial Domains. Rockville, MD, National Institute on Alcoholism and Alcohol Abuse, Research Monograph, 1993

2. Heath AC, Slutske WS, Madden PAF: Gender differences in the genetic contribution to alcoholism risk and to alcohol consumption patterns. Unpublished manuscript, 1995

3. Istvan J, Matarazzo JD: Tobacco, alcohol, and caffeine use: A review of their interrelationships. Psychol Bull 95:301-326, 1984

4. Glassman AH, Helzer JE, Covey LS, Cottler LB, Stetner F, Tip JE, Johnson J: Smoking, smoking cessation, and major depression. JAMA 264:1546-1549, 1990

5. Raaschou-Nielsen E: Smoking habits in twins. Dan Med Bull 7:8288,1960

6. Cederlof R: Twin registries in the study of chronic disease. Acta Med Scand Suppl 523:9-40, 1971

7. Medlund P, Cederlof R, Floderus-Myrhed B, Friberg L, Sorensen S: A new Swedish twin registry: Containing environmental and medical base line data from about 14,000 same-sexed pairs born 1926-58. Acta Med Scand Suppl 600:1-111, 1976

8. Kaprio J, Koskenvuo MD, Langinvainio H, Romanov K, Sarna S, Rose RJ: Genetic influences on use and abuse of alcohol: A study of 5638 aduit Finnish brothers. Alcohol Clin Exp Res 11:349-356, 1987

9. Swan GE, Carmelli D, Rosenman RH. Fabsitz RR, Christian JC: Smoking and alcohol consumption in adult male twins: Genetic heritability and shared environmental influences. J Subst Abuse 2:39-50, 1990

10. Carmelli D, Swan GE, Robinette D, Fabsitz R: Genetic influence on smoking-A study of male twins. N Engl J Med 327:829-883, 1992
11. Heath AC, Martin NG: Genetic models for the natural history of smoking: Evidence for a genetic influence on smoking persistence. Addict Behav 18:19-34, 1993

12. Madden PA, Heath AC, Bucholz KK, Dinwiddie SH, Dunne MP, Martin NG: Genetics and smoking. Paper presented at the 55th annual meeting of The College on Problems of Drug Dependence, Toronto, Canada, June 12-17, 1993

13. Madden PA, Heath AC, Bucholz KK, Dinwiddie SH, Dunne MP, Martin NG: Novelty-seeking and the genetic determinants of smoking initiation and problems related to alcohol use in female twins. Behavior Genetics Association, Sydney, Australia, 23rd Annual Meeting, July 1316,1993

14. Schuckit MA: Ethanol-induced changes in body sway in men at high alcoholism risk. Arch Gen Psychiatry 42:375-379, 1985

15. Lipscomb TR, Carpenter JA, Nathan PE: Static ataxia: A predictor of alcoholism. Br J Addict 74:289-294, 1979

16. Lex BW, Lukas SE, Greenwald NE, Mendelson JH: Alcohol-induced changes in women at risk body sway in women at risk for alcoholism: A pilot study. J Stud Alcohol 49:346-356, 1988

17. McCaul ME, Turkkan JS, Svikis DS, Bigelow GE: Familial density of alcoholism: Effects on psychophysiological responses to ethanol. Alcohol 8:219-222, 1991

18. Schuckit MA: Self-rating of alcohol intoxication by young men with and without family histories of alcoholism. J Stud Alcohol 41:242-249, 1980

19. Schuckit MA: Subjective responses to alcohol in sons of alcoholics and control subjects. Arch Gen Psychiatry 41:879-884, 1984

20. O'Malley SS, Maisto SA: Effects of family drinking history and expectancies on responses to alcohol in men. J Stud Alcohol 46:289-297, 1985

21. Moss HB, Yao JK, Maddock JM: Responses by sons of alcoholic fathers to alcoholic and placebo drinks: Perceived mood, intoxication, and plasma prolactin. Alcohol Clin Exp Res 13:252-257, 1989

22. Savoie TM, Emory EK, Moody-Thomas S: Acute alcohol intoxication in socially drinking female and male offspring of alcoholic fathers. $J$ Stud Alcohol 49:430-435, 1988

23. Bauer LO, Hesselbrock VM: EEG, autonomic and subjective correlates of the risk for alcoholism. J Stud Alcohol 54:577-589, 1993

24. Pollock VE, Teasdale TW, Gabrielli WF, Knop J: Subjective and objective measures of response to alcohol among young men at risk for alcoholism. J Stud Alcohol 47:297-304, 1986

25. Martin NG, Oakeshott JG, Gibson JB, Starmer GA, Perl J, Wilks AV: A twin study of psychomotor and physiological responses to an acute dose of alcohol. Behav Genet 15:305-347, 1985

26. Martin NG, Perl J, Oakeshott JG, Gibson JB, Starmer GA, Wilks AV: A twin study of ethanol metabolism. Behav Genet 15:93-109, 1985

27. Neale MC, Martin NG: The effects of age, sex, and genotype on self-report drunkenness following a challenge dose of alcohol. Behav Genet 19:63-78, 1989

28. Pollock VE: Meta-Analysis of subjective sensitivity to alcohol in sons of alcoholics. Am J Psychiatry 149:1534-1538, 1992

29. Schuckit MA: Low level of response to alcohol as a predictor of future alcoholism. Am J Psychiatry 151:184-189, 1994

30. Schuckit MA: Reaction to alcohol as a predictor of alcoholism. Neuropharmacol Alcohol 15(Suppl. 1, Part A):305A-306A, 1992

31. Tong JE, Knott VJ, McGraw DJ, Leigh G: Alcohol, visual discrimination and heart rate. Q J Stud Alcohol 35:1003-1022, 1974

32. Lyon RJ, Tong JE, Leigh G, Clare G: The influence of alcohol and tobacco on the components of choice reaction time. J Stud Alcohol 36:587-596, 1975

33. Michel C, Battig K: Separate and combined psychophysiological effects of cigarette smoking and alcohol consumption. Psychopharmacology $97: 65-73,1989$

34. Kerr JS, Sherwood N, Hindmarch I: Separate and combined effects of the social drugs on psychomotor performance. Psychopharmacology 104:113-119, 1991

35. De Fiebre CM, Medhurst LJ, Collins AC: Nicotine response and 
nicotinic receptors in long-sleep and short-sleep mice. Alcohol 4:493-501, 1987

36. Collins AC, Marks MJ: Chronic nicotine exposure and brain nicotinic receptors-Influence of genetic factors, in Nordberg $N$, Fuxe $D$, Holmstedt B, Sundwall A (eds): Progress in Brain Research, vol 79. Amsterdam, Elsevier Science Publishers B.V., 1989, pp 137-146

37. De Fiebre CM, Romm E, Collins JT, Draski LJ, Deitrich RA, Collins AC: Responses to cholinergic agonists of rats selectively bred for differential sensitivity to ethanol. Alcohol Clin Exp Res 15:270-276, 1991

38. De Fiebre CM, Collins AC: Classical genetic analyses of responses to nicotine and ethanol in crosses derived from long- and short-sleep mice. J Pharmacol Exp Ther 261:173-180, 1992

39. De Fiebre CM, Marks MJ, Collins AC: Ethanol-nicotine interactions in long-sleep and short-sleep mice. Alcohol 7:249-257, 1990

40. De Fiebre CM, Collins AC: Behavioral desensitization to nicotine is enhanced differentially by ethanol in long-sleep and short-sleep mice. Alcohol 6:45-51, 1989

41. Jardine R, Martin NG, Henderson AS: Genetic covariation between neuroticism and the symptoms of anxiety and depression. Genet Epidemiol 1:89-107, 1984

42. Heath AC, Madden PAF, Slutske WS, Martin NG: Personality and the inheritance of smoking behavior: A genetic perspective. Behav Genet 25:103-117, 1995

43. Joreskog KG, Sorbom D: PRELIS: A Preprocessor for LISREL, ed

2. Mooresville, IN, Scientific Software, 1988

44. Joreskog KG, Sorbom D: LISREL7: A Guide to the Program and Applications, ed 2. Chicago, IL, SPSS, Inc., 1989

45. Bollen KA: Structural Equations with Latent Variables. New York,
John Wiley \& Sons, 1989

46. McGue M, Wette R, Rao DC: Evaluation of path analysis through computer simulation: Effect of incorrectly assuming independent distribution of familial correlations. Genet Epidemiol 1:255-269, 1984

47. Neale MC, Cardon LR (eds): Multivariate analysis, in Methods for Genetic Studies of Twins and Families. Boston, MA: Kluwer Academic Publishers, 1992, pp 231-259

48. DeWit H, McCracken SG: Ethanol self-administration in males with and without an alcoholic first-degree relative. Alcohol Clin Exp Res 14:63-70, 1990

49. Vogel-Sprott M, Chipperfield B: Family history of problem drinking among young male social drinkers: Behavioral effects of alcohol. J Stud Alcohol 48:430-436, 1987

50. Lipscomb TR, Nathan PE: Blood alcohol discrimination. Arch Gen Psychiatry 37:571-576, 1980

51. Nagoshi CT, Wilson JR: Influence of family alcoholism history on alcohol metabolism, sensitivity, and tolerance. Alcohol Clin Exp Res 11:392-398, 1987

52. Kaplan RF, Hesselbrock VM, O'Connor S, Depalma N: Behavioral and EEG responses to alcohol in nonalcoholic men with a family history of alcoholism. Prog Neuro-Psychopharmacol Biol Psychiatry 12:873-885, 1988

53. Newlin DB, Thomson JB: Alcohol challenge with sons of alcoholics: A critical review and analysis. Psychol Bull 108:383-402, 1990

54. Pomerleau OF, Collins AC, Shiffman S, Pomerleau CS: Why some people smoke and others do not: New perspectives. J Consult Clin Psychol 61:723-731, 1993 